\title{
Ultrasound for Detection of Ascites and for Guidance of the Paracentesis Procedure: Technique and Review of the Literature
}

\author{
Josh Ennis ${ }^{1 *}$, Geoffrey Schultz ${ }^{2}$, Phillips Perera ${ }^{1}$, Sarah Williams ${ }^{1}$, \\ Laleh Gharahbaghian', Diku Mandavia ${ }^{3}$ \\ ${ }^{1}$ Division of Emergency Medicine, Stanford University Medical Center, Stanford, USA \\ ${ }^{2}$ Emergency Professional Services, Phoenix, USA \\ ${ }^{3}$ Department of Emergency Medicine, LAC+USC Medical Center, Los Angeles, USA \\ Email: ${ }^{*}$ joshennismd@gmail.com
}

Received 8 September 2014; revised 6 October 2014; accepted 5 November 2014

Academic Editor: Darrell Sutijono, Kaiser Santa Clara Medical Center, USA

Copyright (C 2014 by authors and Scientific Research Publishing Inc.

This work is licensed under the Creative Commons Attribution International License (CC BY).

http://creativecommons.org/licenses/by/4.0/

c) (i) Open Access

\section{Abstract}

Objective: To review the use of ultrasound (US) for the detection of free intraperitoneal fluid (ascites) and for the procedural guidance of the paracentesis procedure. Methods: Two clinical vignettes are presented to review the pertinent diagnostic, management and safety considerations associated with paracentesis. First, US techniques used for the identification of ascites and in the quantification of fluid pockets amenable to aspiration will be discussed. Next, the actual steps required for the performance of US-guided paracentesis will be covered. A review and analysis of the most current literature regarding US and paracentesis then follows. Conclusion: Current literature favors US-guided paracentesis over the traditional blind technique with a significant reduction in both the rate of unsuccessful aspiration of fluid and in the bleeding complications related to this procedure. Use of US for both the diagnostic and therapeutic management of ascites should be advocated as an essential skill for physicians and other health care providers caring for these patients.

\section{Keywords}

Paracentesis, Ascites, Ultrasound, Review, Procedure

\footnotetext{
${ }^{*}$ Corresponding author.
} 


\section{Introduction}

Ascites is a common end point of multiple disease states that lead to leakage of fluid into the peritoneal cavity. The most common etiology for ascites is liver dysfunction and hepatic cirrhosis, which lead to transudation of fluid into the peritoneum as a result of high portal venous pressures. Other significant causes of ascites include renal failure and cardiac failure. In developing nations, severe hypoalbuminemia from malnutrition is a frequent etiology. Furthermore, ascites may often be seen in cases of primary abdominal and gynecological malignancies, as well as in cancers that are metastatic to the liver. The accumulation of ascites results in progressive abdominal distention and significant patient discomfort. As abdominal pressure increases, elevation of the diaphragms leads to reduced lung volumes and lowered functional respiratory reserve. This often causes shortness of breath, especially when the patient is recumbent. While medical therapies, such as reduction in salt intake and diuretics are commonly used to treat ascites, a significant subset of medically managed patients will not improve [1]. Large volume paracentesis may be emergently indicated to alleviate painful abdominal distention, dyspnea and resultant respiratory compromise, especially in diuretic resistant patients and those unable to comply with their medical regimen [2].

The presence of ascites also predisposes to spontaneous bacterial peritonitis (SBP), a potentially life threatening infection caused by transmigration of bacteria into the peritoneal fluid. Unfortunately, physician's clinical impression has a limited sensitivity for this diagnosis. One study demonstrated a sensitivity of $76 \%$ and specificity of 34\% for the physician impression in accurately detecting SBP [3]. The timely performance of paracentesis is critical to making the initial diagnosis of SBP, allowing ascites fluid to be obtained for definitive testing. Appropriate antibiotic therapy can then be selected based on the results of these tests [4].

\section{History}

Traditionally, paracentesis was performed blindly using standard anatomical landmarks [2]. As the etiology of ascites in most patients is liver cirrhosis with associated portal hypertension, the liver typically becomes smaller and the spleen larger as disease progresses. Therefore, some physicians favor the right paracolic gutter approach. However, the patient with ascites may have atypical anatomy, due to varying dimensions of the spleen and liver in different pathology states. Therefore, others refer to the left lower quadrant (LLQ) as the typical puncture point [5]. The midline linea alba technique has also been historically employed with success [6]. While the blind landmark technique continues to be used effectively in current medical practice by experienced operators, it is associated with an increased rate of unsuccessful aspiration attempts [7] [8]. In addition, the risk of complications, particularly bleeding into the abdomen, is higher with this technique [9] [10]. Finally, ascites pockets amenable to drainage may only be present in localized regions of the abdomen and not in the standard areas used for the landmark technique [11].

The utility of bedside abdominal US for the identification of free intra-peritoneal fluid and drainage of ascites has been recognized since the 1980's [12]. One study demonstrated that as little as 100 cc of fluid could be seen on US in a cadaver model [13]. Further use of US for the assessment of free intra-peritoneal fluid has developed from research in trauma patients with the Focused Assessment with Sonography in Trauma ("FAST") exam (Figure 1). As little as 150 cc of fluid may be detected using the FAST suprapubic view [14]. Larger volumes of 500 - 600 cc may be more reliably identified, as this amount is typically required for a positive right upper quadrant FAST view [15]. Clinicians have extrapolated from these studies that free ascites fluid could also be rapidly detected through the use of the standard FAST views. In addition, US views of the right and left paracolic gutters are generally included for the specific detection of ascites. The use of US to diagnose ascites has been supported by the fact that physical examination findings, like shifting dullness and a palpable fluid wave, have only an intermediate diagnostic sensitivity (70\% - 80\%) due to the larger volumes of ascites required for a reliable positive examination [16] [17].

Today, many clinicians have now integrated bedside US into the initial assessment of patients with suspected ascites [18]. The majority of residency training programs in Emergency Medicine now teach the use of US to improve diagnostic accuracy [19]. This has set the stage for the increased use of bedside US to improve diagnostic accuracy for ascites, especially in cases of smaller volumes of fluid.

\section{Ultrasound for Paracentesis}

As bedside US has become more widely used in the assessment of ascites, many clinicians have also routinely 


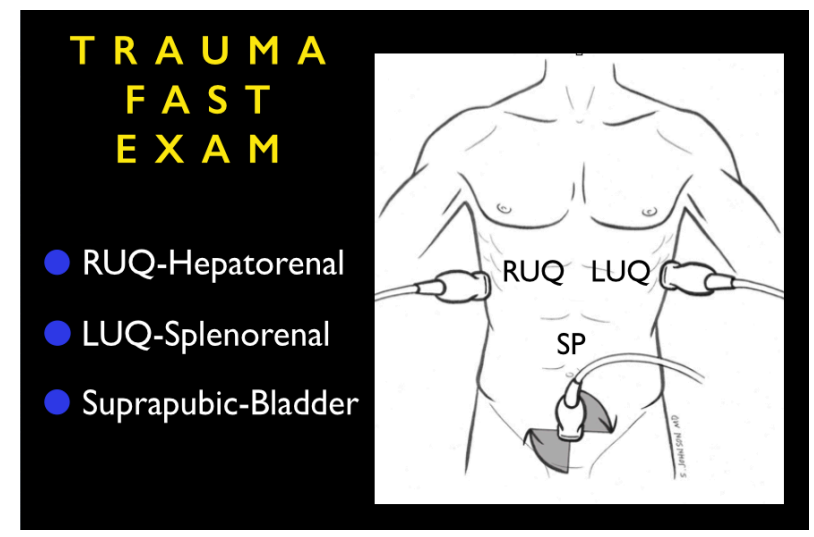

Figure 1. Trauma FAST exam views (abdominal and pelvic).

integrated US guidance into the paracentesis procedure. The anatomy of the liver and spleen can be further defined, so as to avoid these structures during the procedure (Figure 2, Figure 3).

The suprapubic region in males and females should also be closely examined, as this is the location where ascites may first be seen (Figures 4-6). Next, the location of the largest pockets of ascites can be individually identified in each patient. One study looked specifically at the volumes of ascites pockets using the smallest fluid depth measurement ("SFD"- the distance from the most superficial bowel loop to the abdominal wall). This demonstrated a correlation of SFD with the volume of drained paracentesis fluid [11]. The authors found that for every $1 \mathrm{~cm}$ increase in the SFD, there was a correlation in the amount of drained fluid by 1 liter.

Next, the abdominal wall can be evaluated in areas where needle placement is being considered. This is helpful in finding the thinnest regions, as patients with ascites may have significant edema with resultant thickening of the abdominal wall. The length of the needle that will best traverse the wall can be determined by this measurement. Finally, vascular structures within this tissue can be identified and avoided, particularly the inferior epigastric vessels and any collateral structures that may have developed due to portal hypertension [20]. With the knowledge gained by bedside US, the procedure can then be more safely performed [21].

Available evidence now supports the use of ultrasound prior to the paracentesis procedure for the purposes of site planning and for the avoidance of procedural complications, including bowel injury, laceration of vascular structures and failed acquisition of ascites fluid. While experienced physicians may have a higher success rate and lower complication rate using the blind technique [7], individuals performing paracentesis on a less than frequent basis likely benefit more from US guidance. This becomes even more important in patients with lower volumes of ascites. One case series found the absence of drainable fluid collections at the traditional LLQ site of blind paracentesis in patients with progressively lower volumes of ascites [22]. They also noted potentially complicating loops of air-filled bowel interposed at this site between the abdominal wall and drainable collections of fluid.

\section{Report of Cases}

\subsection{Case 1}

A 60-year-old male with known alcoholic cirrhosis presented to the Emergency Department (ED) with the chief complaint of increasing abdominal girth, abdominal discomfort and progressive shortness of breath. His vital signs were: blood pressure of 90/50 mm $\mathrm{Hg}$, heart rate of 92 beats per minute, temperature of 98.7 degrees $\mathrm{F}$, respiratory rate of 22 per minute and oxygen saturation of $93 \%$. On physical examination, he had impressive abdominal distention and was noted to be in respiratory distress when lying recumbent. A fluid wave was thought to be present on abdominal examination. Mild subjective tenderness to palpation was noted in all abdominal quadrants. Notably absent on abdominal examination were rebound tenderness and guarding. A review of the patient's medical record demonstrated previous visits for similar complaints that were successfully managed with large volume paracentesis.

An US machine was brought to the bedside and confirmed the presence of ascites and determined the optimal location for the paracentesis procedure. After obtaining informed consent and performing a time-out prior to the 


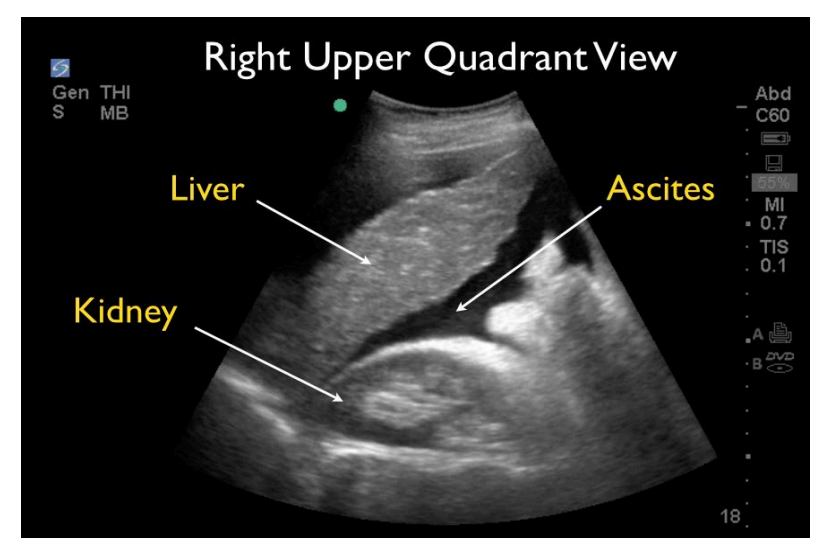

Figure 2. Right upper quadrant ultrasound view.

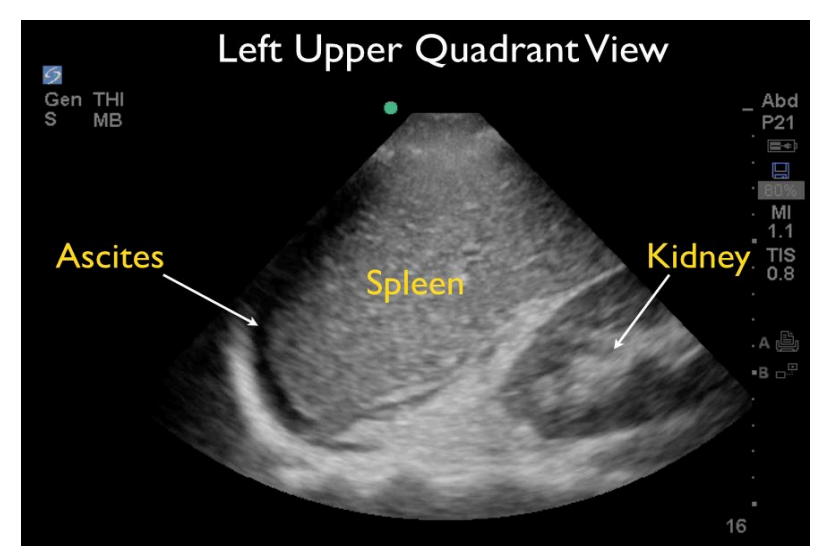

Figure 3. Left upper quadrant ultrasound view.

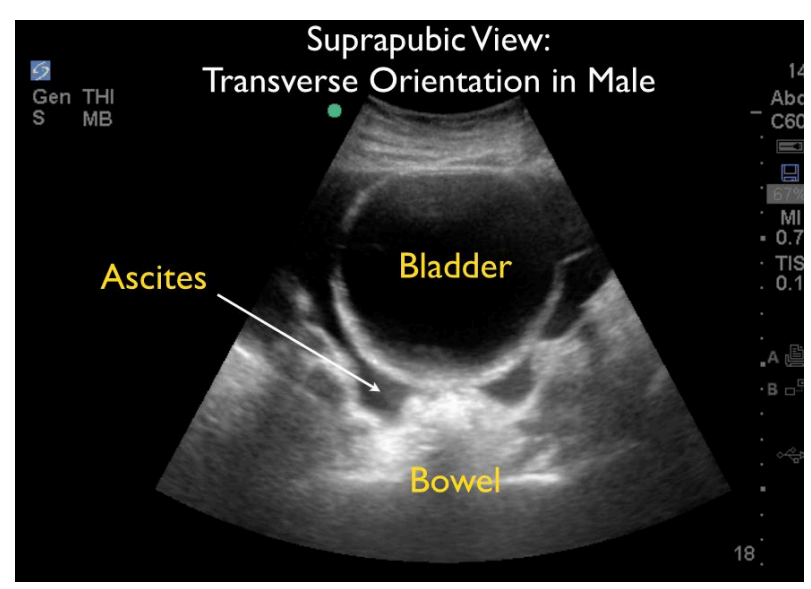

Figure 4. Suprapubic ultrasound view with free fluid.

procedure, 5 liters of clear yellow fluid were successfully drained from the LLQ area. The patient felt much better. He received albumin intravenously and was subsequently discharged from the ED for close follow-up in the Gastroenterology clinic.

\subsection{Case 2}

A 45-year-old woman with previous history of colorectal adenocarcinoma, status post resection and chemotherapy, presented to the ED with abdominal distention and pain. The patient's vital signs were: blood pressure 


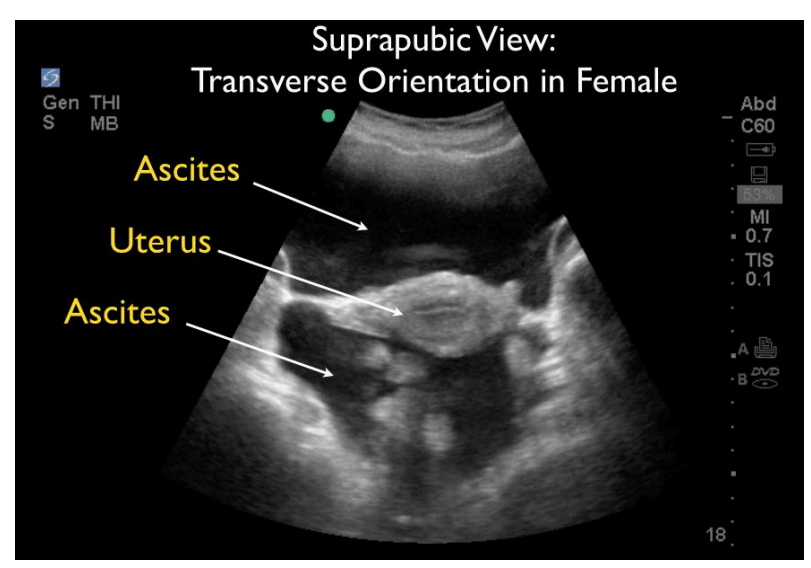

Figure 5. Suprapubic ultrasound view with free fluid.

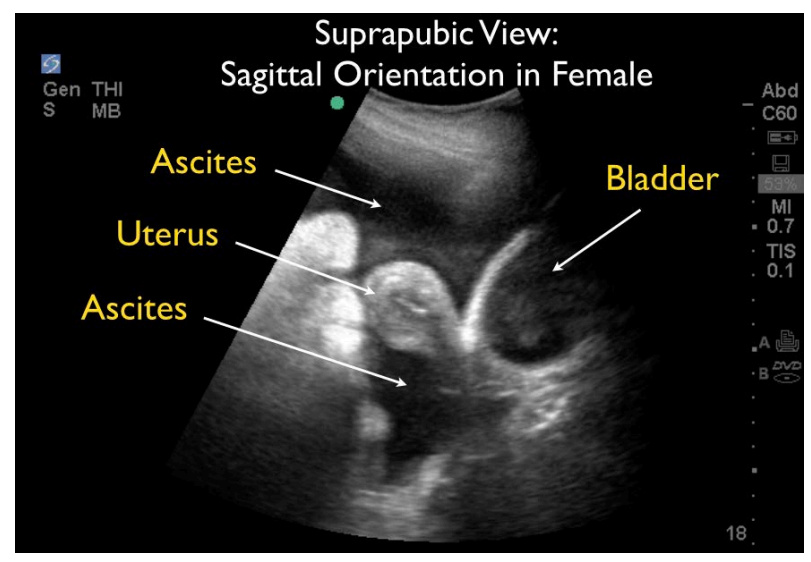

Figure 6. Suprapubic ultrasound view with free fluid.

110/60 mm Hg, a heart rate 112 beats per minute, an oral temperature of 101.9 degrees F, a respiratory rate of 20 per minute and an oxygen saturation of $99 \%$ on room air. The presence of diffuse rebound tenderness on examination prompted the treating physicians to perform a CT scan of the abdomen. This revealed a small amount of free intraperitoneal fluid and solid lesions within the liver concerning for metastatic disease.

Given the clinical concern for SBP in the setting of ascites, fever and abdominal pain, a diagnostic paracentesis was clinically indicated. Because the patient had only mild abdominal distention on physical examination and a small amount of free fluid on CT scan, bedside US was utilized. US identified a fluid collection in the right lower quadrant area, which was then successfully drained. Collection of fluid may not have been possible via the traditional blind technique, due to the small amount of fluid present. Cell count, gram stain and cultures were ordered and the patient was treated empirically with intravenous antibiotics and subsequently admitted to the hospital.

\subsection{Use of Ultrasound}

These clinical cases highlight the importance of bedside US in the diagnosis and management of patients with both worsening ascites and SBP. Bedside US allows for the rapid diagnosis and subsequent safe drainage of ascites, even when fluid pockets are small [23].

\section{Indications and Contraindications for the Paracentesis Procedure}

According to the World Gastroenterology Organization, diagnostic paracentesis is indicated in all patients with new onset ascites, and in those patients with known ascites whose clinical condition deteriorates (4). More specifically, patients with known or new onset ascites and clinical signs of SBP require immediate paracentesis [3] [23]. Therapeutic, or large volume paracentesis, is indicated for ascites refractory to traditional medical therapy 
[24].

Generally, the only absolute contraindications to the paracentesis procedure are clinically evident fibrinolysis or disseminated intravascular coagulation [25]. In other cases, paracentesis, especially with US guidance, does not generally need to be preceded by laboratory evaluation for coagulopathy or thrombocytopenia. Studies have found that coagulopathy and thrombocytopenia are not linked to a higher risk of bleeding complications [26]. Transfusion with fresh frozen plasma or platelets in an effort to correct laboratory abnormalities is therefore not usually indicated prior to paracentesis. Interestingly, advanced renal disease is more commonly associated with paracentesis related hemorrhage [27] [28]. Patients with advanced renal disease must therefore have careful consideration prior to this procedure and US guided paracentesis should generally be used in these patients. Relative contraindications to paracentesis include the uncooperative patient, skin infection at the site of puncture, pregnancy, surgical scars (where the bowel may be entrapped close to the abdominal wall) and severe bowel distention [29].

\section{Indications and Contraindications for the Paracentesis Procedure}

\subsection{Bladder Decompression}

Fully emptying the bladder, either by having the patient void or placing a Foley catheter, is indicated prior to paracentesis. This will avoid inadvertent bladder puncture during the procedure. US determination of bladder volume can also be employed prior to the procedure to determine the need for further bladder decompression.

\subsection{Informed Consent and Preparation}

Prior to paracentesis, informed consent should be obtained from the patient. The relative risks of an US guided paracentesis will be discussed below and the clinician can relay this information to the patient. Next, a time-out procedure should be performed, during which patient identity, clinical information, proposed invasive procedure and operative site should be confirmed, together with the patient's nurse. The clinician should then implement full-barrier sterile precautions. This includes carefully washing the hands and putting on a mask, sterile gown and gloves. The patient is prepared by using several chlorhexidine swabs to fully cleanse the proposed puncture area. Alternatively, betadine may be used if chlorhexidine is unavailable. A full-size sterile sheet should be placed over the patient, with only the puncture site uncovered [30].

\subsection{Patient Positioning}

Next, optimal positioning of the patient for the paracentesis procedure is important to success. Patients with a significant volume of ascites will often have difficulty lying completely supine during the procedure, due to orthopnea from increased upward pressure on the diaphragms. Patients should be placed with the head of the bed raised to the point where maximal comfort is achieved. Optimally, this will be at about 30 degrees, with the head up. This maneuver will also move the ascites fluid to the dependent areas of the abdomen, making it easier to obtain fluid from the paracolic or midline linea alba approaches. If a lateral paracolic approach is being used, positioning the patient in a modified lateral decubitus position can be helpful by increasing the relative size of the ascites fluid pocket on a specific side. The side selected for the paracentesis should be dependent. Placing pressure with a hand on the contralateral side of the abdomen may also increase the size of a dependent fluid pocket. If the patient is able, they may assist in this maneuver.

\subsection{Initial Probe Choice and Fluid Localization}

Prior to the paracentesis procedure, US should be used to identify ascites and to search for the largest pockets of fluid. A $3 \mathrm{MHz}$ curvilinear or phased array probe is recommended for this initial assessment. This lower frequency probe has a longer wavelength, allowing the clinician to look deeper into the patient's abdomen and pelvis, giving a more global view. A modified FAST exam can be employed for a general assessment of the abdomen and pelvis. The positions for placement of the probe to assess the traditional needle puncture areas for paracentesis are demonstrated in Figure 7. Ascites will typically appear dark (anechoic) on US. Loops of bowel with mesentery may be seen floating within the ascites fluid, and will typically move as the patient changes position or takes a breath (Figure 8, Figure 9). Complicated ascites may be identified by the characteristic appearance of 


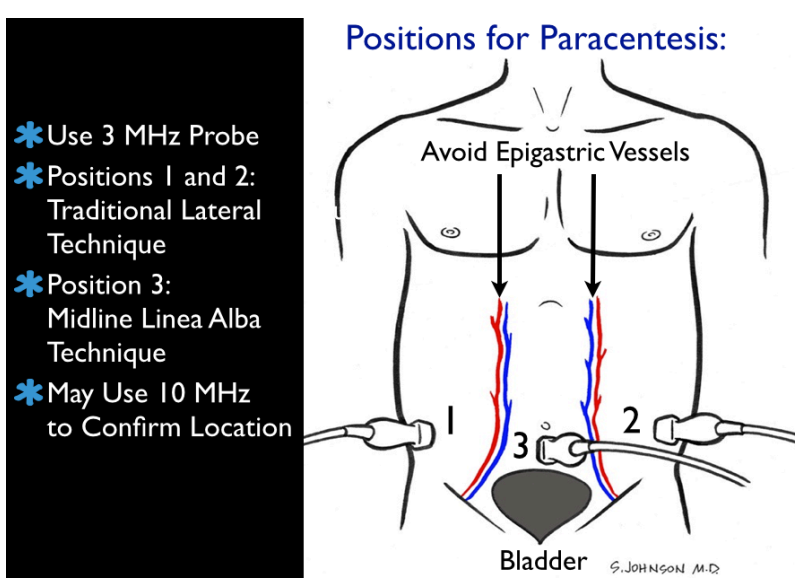

Figure 7. Paracentesis probe placement.

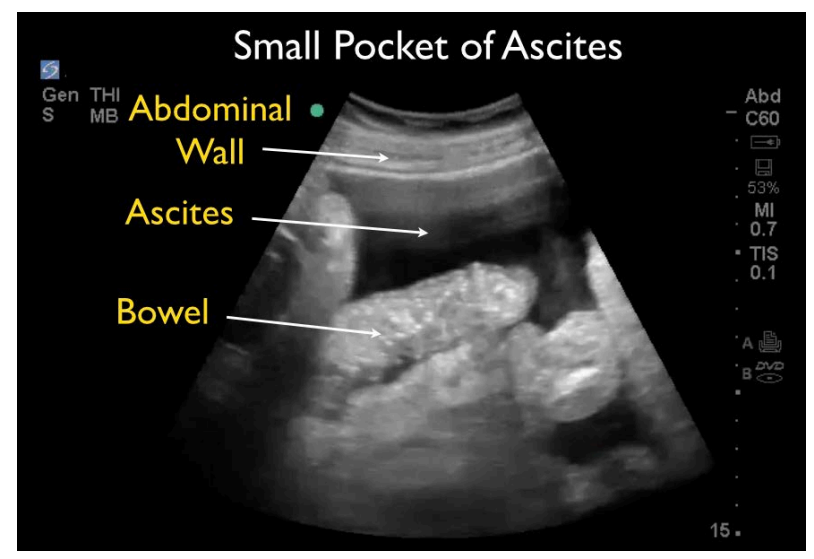

Figure 8. Ultrasound: Small pocket of ascites.

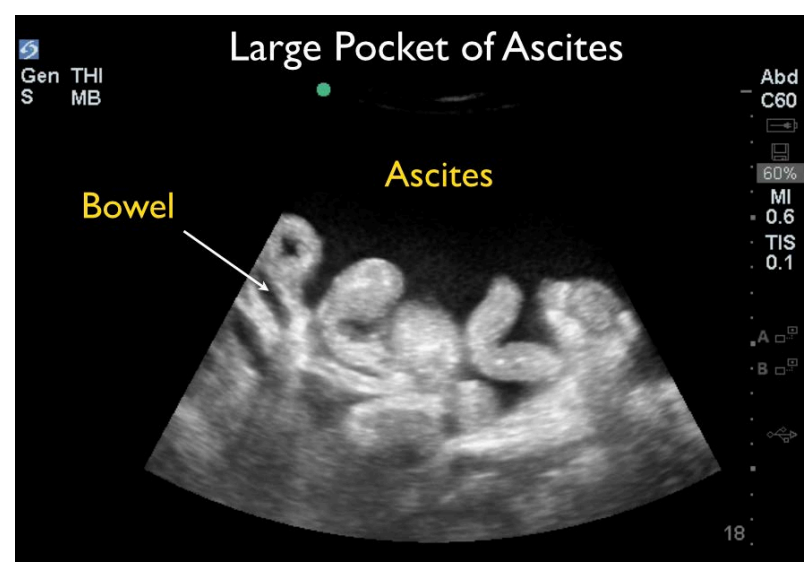

Figure 9. Ultrasound: Large pocket of ascites.

the fluid on US. Hemoperitoneum may be indicated by a complex appearance of the ascites fluid, with internal echoes of blood interspersed in the fluid (Figure 10). Loculations, typically caused by fibrin stranding, may occasionally be present, and can be identified by a bright (hyperechoic) appearance on US (Figure 11). Smaller loculated areas of ascites should be avoided for paracentesis, especially if there are other areas of free ascites present.

A determination is then made regarding the amount of ascites present and the likelihood that the fluid can be safely aspirated. The Word Gastroenterology Organization states that at least $20 \mathrm{~mL}$ of fluid is necessary for di- 


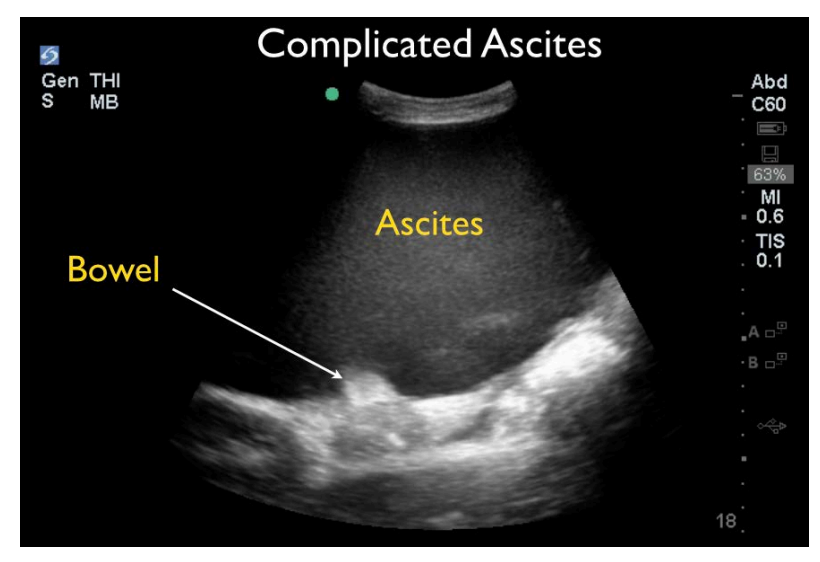

Figure 10. Ultrasound image of echogenic ascites found in complicated ascites with hemoperitoneum.

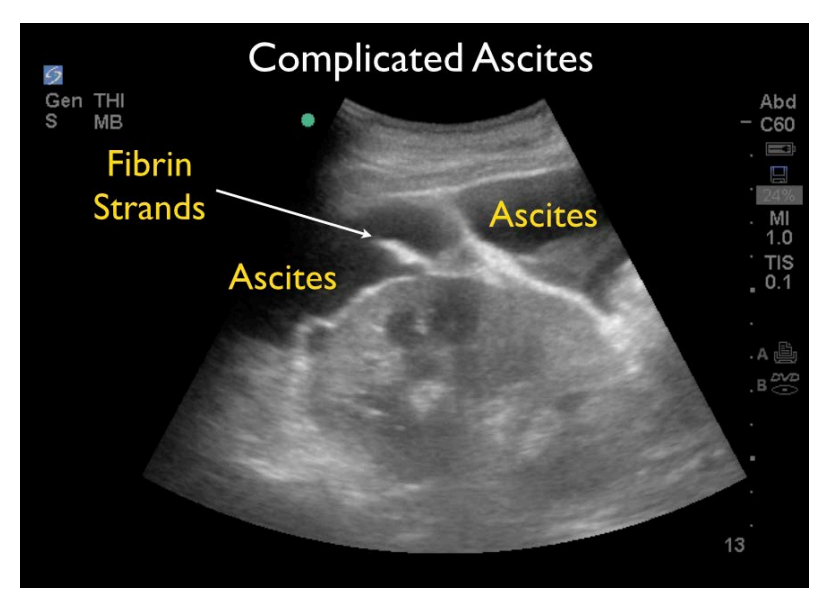

Figure 11. Ultrasound image of complicated ascites with fibrin strands.

agnostic paracentesis [4]. Other studies suggest 50 - 100 cc as the minimum amount of fluid needed [22]. As mentioned above, one can gauge a rough estimate of the quantity of ascites fluid by measuring the size of the pockets of fluid [11].

\subsection{Identification of Puncture Site}

The next step is to look more closely at the proposed puncture site using a high frequency $10 \mathrm{MHz}$ linear probe. This probe has a correspondingly shorter wavelength that allows for more detailed imaging of superficial body structures, specifically the abdominal wall (Figure 12). First, measure the abdominal wall, as it may be edematous and correspondingly thickened in patients with ascites (Figure 13). The best location for placement of the paracentesis needle is generally where the abdominal wall is thinnest. As mentioned above, the midline linea alba was previously thought to be the location with thinner abdominal wall measurements. However, the lateral paracolic gutter areas may actually be the sites with the least wall thickness, especially in patients with a high BMI.

\subsection{Evaluation of Vascular Structures and the "Ascites Safety Zone"}

Finally, the high frequency linear probe should be used to identify critical vascular structures within the abdominal wall. The anatomical location of the inferior epigastric artery and vein has considerable variation between patients. Furthermore, its location below the rectus muscle makes it effectively non-compressible if punctured [31] [32]. Patients with portal hypertension from cirrhosis may also have other vascular collaterals present within the abdominal wall that must be avoided. Historically, the midline line alba approach was promoted due to the 


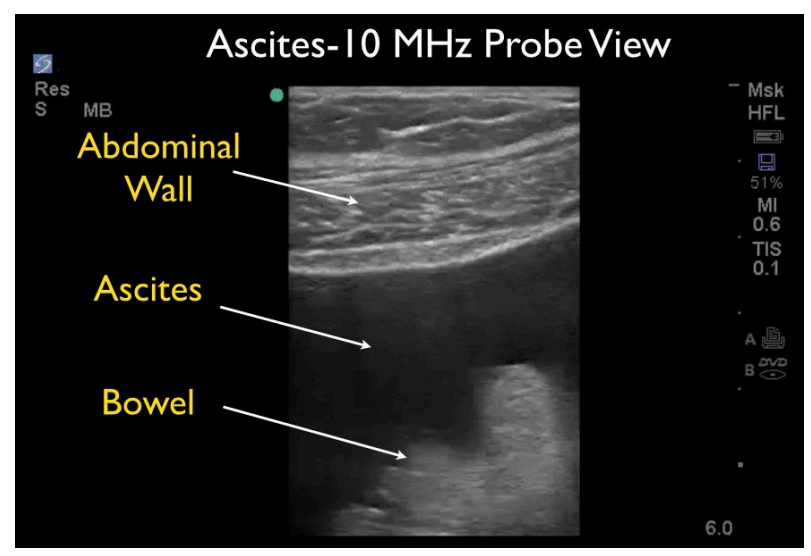

Figure 12. Ultrasound of ascites with linear probe.

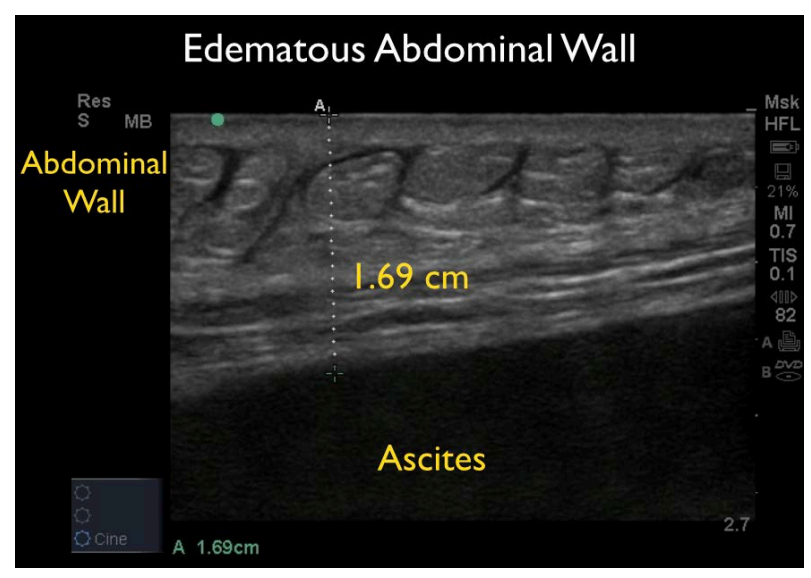

Figure 13. Ultrasound of abdominal wall with linear probe.

hypothesis that this area was relatively avascular. However, one study found that multiple venous structures may course through this region in patients with portal hypertension [33]. Therefore, accurate identification of abdominal wall vascular structures is critical in avoiding a hemorrhagic procedural complication. Vascular structures can first be identified using B-mode US, or grey scale imaging, by looking for the typical rounded appearance of artery and vein (Figure 14). Color Doppler US can then be used to confirm the presence of vascular structures (Figure 15). The optimal puncture site for the procedure should avoid any identified vascular structures in the abdominal wall. One can then determine the "ascites safety zone", or the fluid-filled region below the abdominal wall and above the bowel and mesentery, into which a needle can be safely placed (Figure 16).

\subsection{Static vs. Dynamic Procedural Technique}

There are two general techniques for incorporating US into the paracentesis procedure. The first is the static technique. This technique utilizes bedside US prior to sterile preparation to mark the ideal location for paracentesis. The patient should be placed in the optimal position, the ideal location for the needle puncture determined and a marking pen used to identify the site. The US probe is then removed, the patient prepared and the needle inserted at the marked site. The second technique is the dynamic technique. This utilizes a sterile probe cover so that US may be used to guide and monitor needle insertion into the peritoneal space in real time. The dynamic technique is the currently recommended technique for central venous catheter insertion.

The authors generally recommend the static technique for paracentesis. However, conversion to the dynamic technique is recommended if difficulty is encountered during the procedure. Either the 3 or $10 \mathrm{MHz}$ probes can be used for the dynamic technique (Figure 17). The $10 \mathrm{MHz}$ probe has an additional benefit, as it allows the clinician to directly observe the needle as it passes through the abdominal wall into the ascites fluid (Figure 18). 


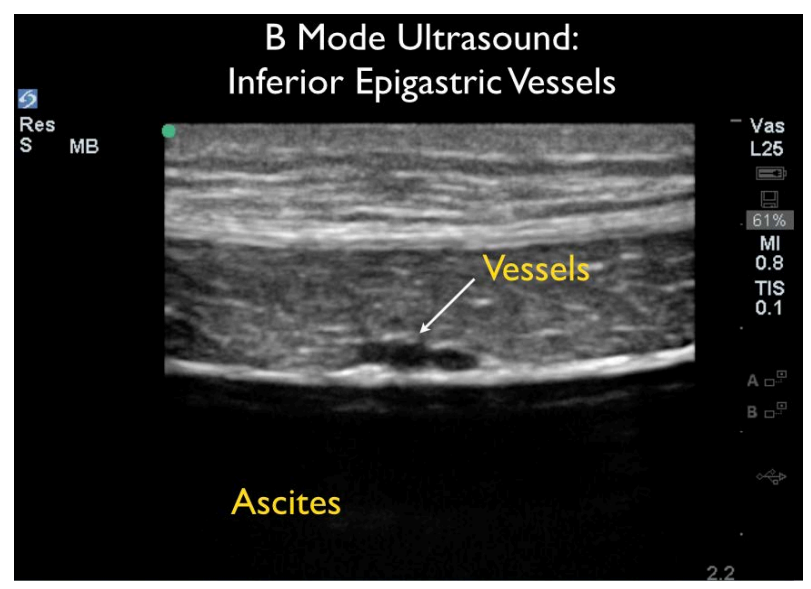

Figure 14. Inferior epigastric vessels (B-mode).

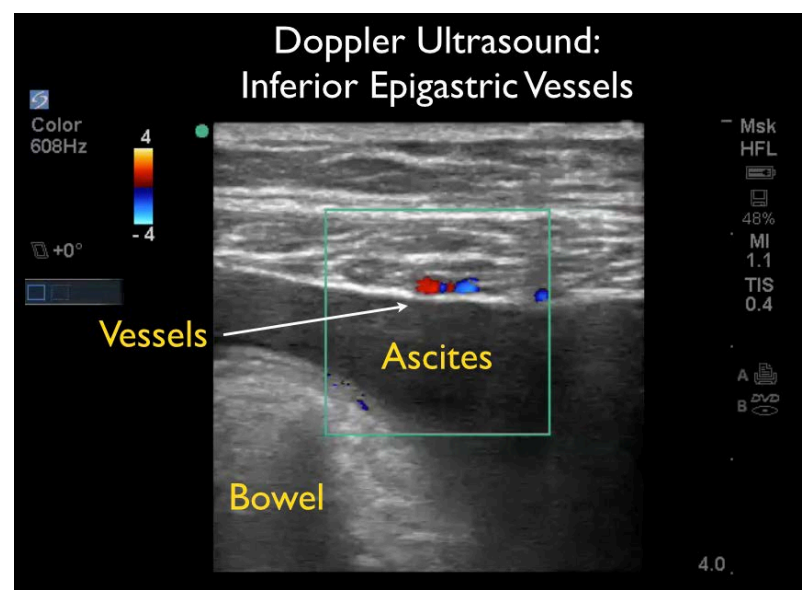

Figure 15. Inferior epigastric vessels with color doppler.

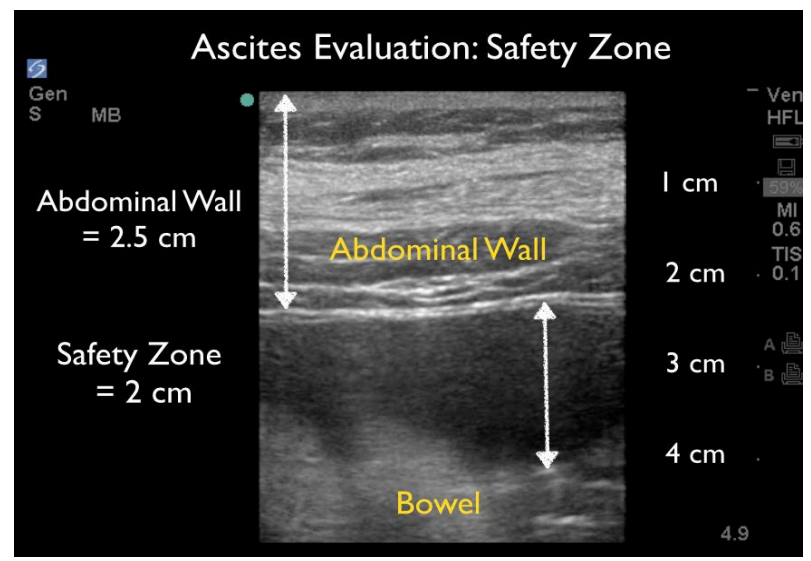

Figure 16. Evaluation for ascites "safety zone", the ascites filled space that lies between peritoneal lining of abdominal wall and the bowel.

\subsection{Local Anesthesia}

Once the site is identified and both the patient and clinician are properly prepared for the sterile procedure, local anesthesia should be applied liberally along the planned trajectory of the needle. Lidocaine is typically used, al- 


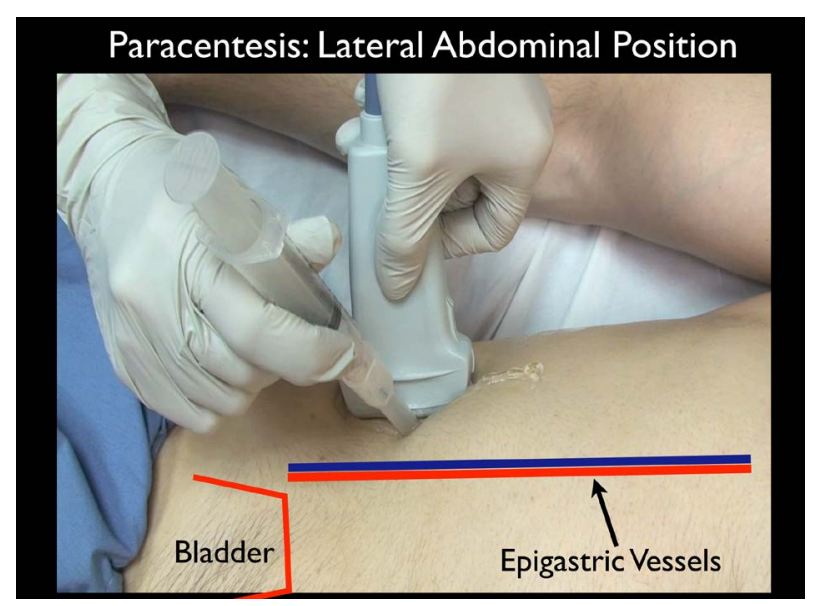

Figure 17. Paracentesis lateral approach.

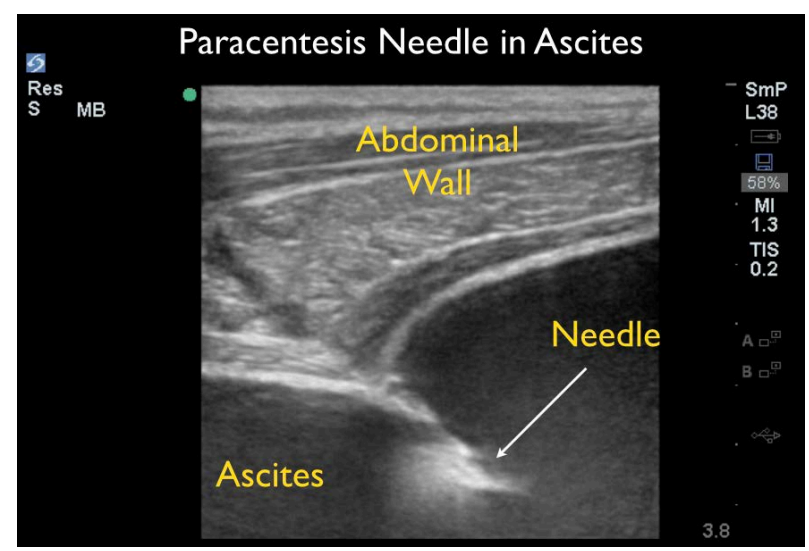

Figure 18. Ultrasound image of needle placed through the abdominal wall into ascites.

though bupivacaine may be substituted for its longer duration of action. A smaller gauge needle may be used for the initial skin anesthesia, while a larger gauge needle is helpful to reach the deeper parts of the abdominal wall and the peritoneum. Critical to achieving good anesthesia is ample placement of the anesthetic agent directly at the peritoneal lining, as the many nerve fibers that innervate this structure make it especially sensitive to pain.

\subsection{Needle Choice}

Different needles may be utilized for peritoneal fluid drainage. A large gauge angiocatheter or needle may be used to aspirate smaller volumes of fluid, as in the case of diagnostic paracentesis for suspected SBP. However, it is necessary to ensure the needle has adequate length, as the abdominal wall often becomes more edematous and thick in patients with ascites. Angiocatheters and shorter needles may not have the required length to successfully aspirate fluid. In addition, it is not uncommon for an angiocatheter to pull the abdominal wall down into the abdominal cavity with resultant obstruction of the needle tip, leading to unsuccessful aspiration (Figure 19). Successful performance of the paracentesis procedure often benefits from the use of the larger needles specifically designed for this purpose. The standard paracentesis and thoracentesis kits are equipped with a larger gauge Caldwell type needle. This needle has the longer length often needed for a successful procedure (Figure 20). One study demonstrated an increased success rate for the aspiration of ascites using a Caldwell type needle, as compared to a standard angiocatheter [34].

\subsection{Technique for Needle Placement}

Following generous anesthesia, a scalpel is used to nick the skin to open a tract for the longer Caldwell type 


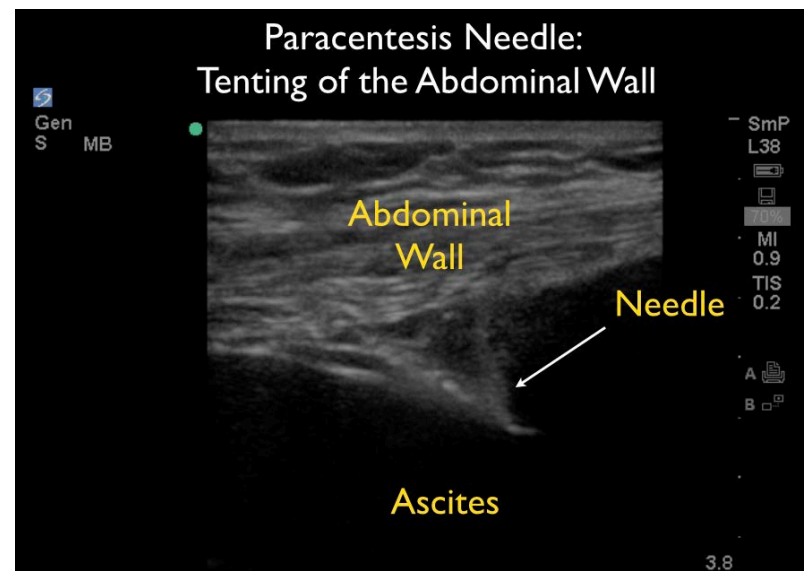

Figure 19. Ultrasound image of abdominal wall peritoneal tenting with needle pumcture.

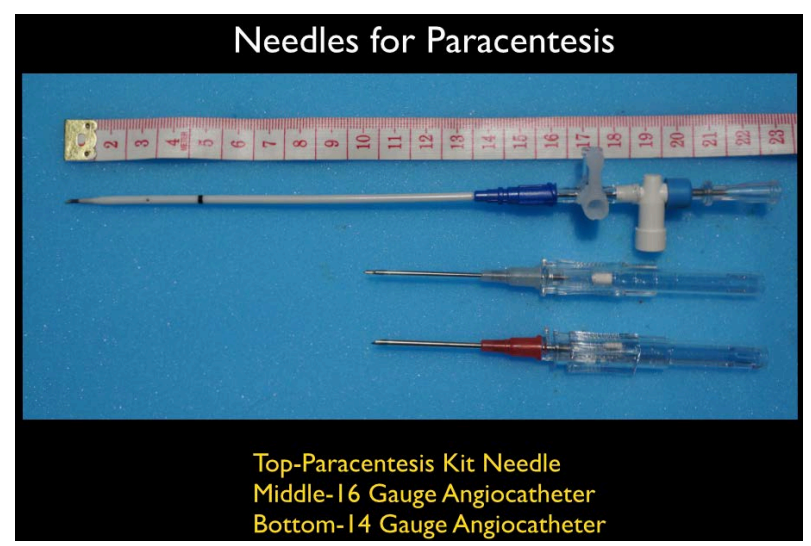

Figure 20. Paracentesis needles.

needle. The paracentesis needle is then advanced with constant negative pressure applied to the syringe. Once the tip of the needle traverses the abdominal wall and enters the peritoneum, ascites fluid will return. Fluid for usual studies can then be immediately collected from this initial aspirate. In the case of a therapeutic paracentesis, the plastic catheter is then gently advanced into the ascites fluid, as the metal trocar is simultaneously removed. This maneuver leaves only a plastic catheter in the peritoneal cavity for continued fluid drainage, decreasing the risk of inadvertent puncture of bowel or solid organ by the metal needle or trocar. Once steady flow of ascites fluid is confirmed from the plastic catheter, the catheter can be attached to a vacutainer bottle for continued removal of fluid.

\subsection{Avoiding Ascites Leakage Following the Procedure}

Additional strategies for needle placement during intra-peritoneal access have been developed to decrease the incidence of a continued leak of ascites following paracentesis puncture [35]. The simplest strategy for needle insertion is to insert it with a straight trajectory, perpendicular to the abdominal wall. An alternative to this method is the z-technique. The z-technique of needle insertion is thought to minimize continued fluid drainage via the catheter tract. Continued drainage can be quite uncomfortable for the patient. More concerning, this indicates a patent tract that may predispose to local and intra-peritoneal infection from adjacent skin flora. To minimize post-procedural drainage, the desired site of insertion is first selected with US. The skin at the desired site is then moved superiorly by applying tension with a sterile, gloved hand. While the skin is under tension, the needle is inserted in a perpendicular orientation through the skin, subcutaneous tissues and abdominal wall tissue. Once the fluid is obtained, tension on the skin is released. Following completion of the procedure and removal of the catheter, the overlying skin will return to its pre-procedural position at a site some distance from the underlying 
puncture. This can disrupt the tract produced during the procedure and form a type of "skin bandage” that can minimize post-procedural drainage. Post-procedural drainage may occasionally occur following paracentesis despite use of the z-technique. For such instances, the use of local pressure followed by liberal application of a skin adhesive (such as 2-octylcyanoacrylate i.e. Dermabond) to seal the tract at the skin is recommended.

\subsection{Sending Ascites Fluid for Testing}

The volume of fluid removed and specific tests ordered will depend on the indication for the procedure (diagnostic vs. therapeutic). Obtaining ample fluid (20 - $30 \mathrm{cc}$ ) for cell count with differential, gram stain and culture is routinely required following diagnostic paracentesis for the purposes of identifying SBP [25]. As SBP can be difficult to diagnose on clinical evaluation, consideration of these tests is also generally indicated in therapeutic large volume paracentesis. Blood culture bottles should be inoculated at the bedside, optimally with 5 - 10 cc of fluid, immediately after the conclusion of the procedure. Immediate inoculation has been found to be more sensitive in the detection of SBP, as compared to delayed inoculation. In cases of new onset ascites, the fluid may be sent for albumin determination. Comparing the serum to ascites albumin gradient will allow for a better determination of the etiology of the ascites. Additional testing is dependent upon each patient's unique clinical presentation. Other specialized tests include mycobacterial culture, lactate dehydrogenase (LDH), amylase and bilirubin [4].

\subsection{Administration of Albumin}

If a large-volume paracentesis is performed (defined as removal of greater than 5 liters of fluid removed), it is currently recommended that patients receive intravenous albumin in order to avoid potential circulatory collapse and associated complications [2].

\subsection{Interpretation of Ascites Results and Empiric Treatment}

An ascites polymorphonuclear (PMN) leukocyte count of $\geq 250$ cells $/ \mathrm{mm}^{3}$ is diagnostic of SBP, and in the appropriate clinical setting should be treated as such [25]. The PMN response, however, may occasionally be delayed, and a positive gram stain and culture may result from ascites sampled prior to the development of the benchmark of $\geq 250 \mathrm{PMNs} / \mathrm{mm}$ (WGO). In the setting of ascites with abdominal pain, fever, and concern for SBP, a positive gram stain should prompt administration of appropriate antibiotics. For common SBP due to gramnegative enteric organisms, a broad-spectrum antibiotic, such as cefotaxime or ceftriaxone, can be given empirically. Further antibiotic choices can be made on the results of the ascites gram stain and culture.

Gram-negative organisms, such as E. coli, are the most common causes of SBP. However, identification of unusual organisms may prompt additional evaluation and treatment of the patient. For example, identification of polymicrobial species in the ascites fluid may indicate secondary bacterial peritonitis from a bowel perforation, as opposed to typical SBP.

In the evaluation of new onset ascites, the determination of the serum to ascites albumin gradient (SAAG) can be helpful. A level greater than $1.1 \mathrm{~g} / \mathrm{dl}$ correlates well with portal hypertension as the underlying etiology.

\section{Evidence for Abdominal Ultrasound in Decreasing Complications Associated with the Paracentesis Procedure}

Available evidence now supports the use of US prior to the paracentesis procedure for the purposes of site planning and for the avoidance of procedural complications. These are most commonly defined as failed acquisition of ascites fluid, laceration of a vascular structure with hemorrhage into the peritoneal cavity and needle puncture of bowel or bladder. The risk of complications becomes even more important in patients with smaller volumes of ascites. One case series found the absence of drainable fluid collections at the traditional landmark technique LLQ site in patients with progressively lower volumes of ascites [22].

Inability to successfully aspirate ascites fluid is considered a primary limitation of the paracentesis procedure. Some patients may undergo multiple needle punctures without obtaining fluid, potentially increasing the risk of damage to internal organs, such as bowel and bladder. A prospective randomized study comparing US-guided and traditional blind paracentesis looked specifically at the aspiration success rate [36]. Emergency department patients in whom the clinical decision had been made to perform a paracentesis were randomized to one of two 
groups; traditional blind paracentesis vs. US-guided paracentesis. Of the 56 patients randomized to undergo the US-guided procedure, 14 had no demonstrable drainable fluid collection on US and an additional 2 patients had pathology identified on US that precluded paracentesis. Of the 42 patients who had drainable fluid found on US, $95 \%$ had a successful paracentesis. In comparison, only $61 \%$ of those randomized to traditional paracentesis had a successful procedure. In a break from the original study design, 15 of the patients with an initial failed paracentesis in the blind landmark group ultimately had successful aspiration of fluid using US guidance. This study reinforced that US not only allowed for confirmation of ascites amenable to paracentesis, but also demonstrated a higher rate of successful aspiration of fluid over the blind technique. Notably, US guidance allowed for successful aspiration of fluid in patients whom had previously undergone an unsuccessful paracentesis using the blind landmark technique. Other studies have reinforced the utility of US-guided paracentesis to achieve a higher success rate of aspiration of fluid from the abdominal cavity [23].

Perhaps the most feared complication of paracentesis is hemorrhage into the peritoneal cavity. This is a valid concern, given the large size of this space and the inability to achieve hemostasis with direct pressure following inadvertent puncture of an abdominal wall vessel. In addition, liver disease patients undergoing this procedure may have both coagulopathy and thrombocytopenia, potentially increasing the bleeding risk with vessel injury [25] [26]. Even though studies suggest that routine correction of these parameters is not necessary, providers must still exercise caution to avoid this potentially fatal complication. Patients with advanced renal disease are at greatest risk of bleeding [27] [28]. The hemorrhagic complication rate related to paracentesis in most available studies lies between $1 \%$ and $2 \%$ [28]. Please see Table 1 for a summary of the few studies in which the authors have clearly delineated the use of US versus traditional approaches.

Mercaldi and Lanes used claims data to examine the risk of bleeding complications after paracentesis in a series of 69,859 patients. Bleeding complications occurred at a rate of $0.27 \%$ in the US group and $1.25 \%$ in the traditional landmark group. Interestingly, in-hospital death rates between the two groups differed at $3.2 \%$ for the US group and $4.3 \%$ for the traditional group, even though they appeared similarly matched. In a subgroup analysis, those with bleeding complications had an in-hospital mortality rate of $12.9 \%$ versus $3.7 \%$ in those without. This difference resulted in measured increases in both the length of hospital stay and in resultant hospital charges [10].

Bleeding may occur via a number of mechanisms. Laceration of the inferior epigastric vessels is the most frequently documented source. It is theorized, however, that spontaneous rupture of collateralized splachnic cir-

Table 1. Limited summary of studies on paracentesis complications.

\begin{tabular}{|c|c|c|c|c|c|c|}
\hline & \multicolumn{2}{|c|}{ Successful Procedure (i.e. no dry tap) } & \multicolumn{2}{|c|}{ Hemorrhagic } & \multicolumn{2}{|c|}{ Other (e.g. infection, low BP, etc.) } \\
\hline & US-Guided & No US & US-Guided & No US & US-Guided & No US \\
\hline Mallory 1978 Retro $[9]^{*}$ & & & & 4/242 (0.8\%) & & $3 / 242(1.2 \%)$ \\
\hline Runyon 1986 Pro [8] & & & & 4/229 (1.7\%) & & \\
\hline Ross 1989 Retro $[21]^{\dagger}$ & & & & & 3/109 (2.7\%) & \\
\hline Webster 1996 Retro [37] & & & & 4/179 (2.2\%) & & \\
\hline Grabau 2004 Retro $[24]^{* \dagger}$ & & & & $1 / 1100(0.1 \%)$ & & 3/1100 (0.3\%) \\
\hline Nazeer 2005 RCT $[36]^{* *}$ & $40 / 42(95 \%)$ & $27 / 42(64 \%)$ & & & & \\
\hline Cervini 2010 Retro $[42]^{\dagger+}$ & & & & & $4 / 2536(0.2 \%)$ & \\
\hline Patel 2012 Retro $[41]^{* * *}$ & & & 2/723 (0.4\%) & 5/574 (0.9\%) & $3 / 723(0.41 \%)$ & $15 / 574(2.4 \%)$ \\
\hline $\begin{array}{l}\text { Mercaldi } 2013 \\
\text { Retro }[10]^{\dagger+\dagger}\end{array}$ & & & $\begin{array}{c}87 / 31,649 \\
(0.27 \%)\end{array}$ & $\begin{array}{c}478 / 38,210 \\
(1.25 \%)\end{array}$ & & \\
\hline
\end{tabular}

* One with bowel perforation and peritonitis, bowel perforation with abdominal wall abscess, one with catheter fragment left in abdominal wall or cavity; ${ }^{\dagger}$ Hypotension occurred after large volume paracentesis in patients with end-stage malignant ascites. No resuscitation was pursued in 2 patients, while the third responded to IV crystalloid; ${ }^{* \dagger}$ Outpatient referrals for large-volume therapeutic paracentesis in patients without prior abdominal surgeries; ${ }^{* *}$ Numbers modified from original study to include only those patients with enough fluid on ultrasound to safely perform paracentesis; ${ }^{\dagger \dagger}$ Infection rates; ${ }^{* * * *}$ Infection rates; ${ }^{\dagger+}$ Of those with bleeding complications, $12.9 \%$ died in the hospital vs. $3.7 \%$ of patients without bleeding complications. Disclaimer: Studies included are only those with a clear delineation of ultrasound vs. no ultrasound. 
culation (due to decreased abdominal compartment pressure post-paracentesis) may be the underlying pathophysiology in some cases [37] [38]. Choice of puncture site may also play a role. Older case reports suggest that the infra-umbilical midline linea alba approach is in an area where there may be significant intra-abdominal varices or a recanalized umbilical vein. A needle inadvertently placed through these structures may result in potential intra-abdominal hemorrhage [39] [40] [33]. Sakai et al. advocate for an US-first approach before paracentesis, citing the LLQ as the favored site, as mentioned above [5].

Patel and colleagues also showed a lower complication rate with US. They performed a retrospective database analysis on 1297 paracentesis procedures, of which 723 (56\%) were performed with US, and 574 (44\%) without. Hemorrhagic complications were significantly lower in the US group. US was associated with fewer adverse events and lower hospitalization costs. Patients undergoing US-guided paracentesis demonstrated lower rates of post-procedural infection, hematoma and seroma [41].

While infectious complications remain a concern, the rate of association with US guided paracentesis is extremely low. In a retrospective review of over 13,000 US-guided cases performed by the Radiology Department at the Mayo Clinic over a two-year period, researchers found only 14 cases of procedure-related infection. Cases included fine needle aspiration, drain placement, biopsy, pseudoaneurysm injection, thoracentesis and paracentesis. Regarding paracentesis, 2536 cases were performed and only 4 were associated with post-procedural infectious complications [42]. By comparison, 242 consecutive diagnostic paracenteses performed without US resulted in 1 case of generalized peritonitis [9]. These findings suggest that the addition of US does not increase the risk of infectious complications related to paracentesis.

There are limitations in some of the studies in the literature looking at the safety profile and complication rate associated with the paracentesis procedure, both with and without US. Some studies do not include a direct comparison between US guided versus traditional landmark techniques [8] [9] [21] [24] [37]. Many studies are also retrospectively performed, which may limit the interpretation of the data [9] [10] [21] [24] [37] [41] [42]. For example, in these studies, US may have been utilized in cases where difficult access was suspected, a limited amount of ascites was present or in higher risk patients. However, the available data may be used to guide a reasonable risk to benefit analysis for the paracentesis procedure performed both with and without US guidance (Table 1).

\section{Conclusion}

Bedside US is a vital tool in the ED, aiding in both the timely diagnosis of potentially life-threatening conditions and in the safe and effective treatment of these patients. The studies reviewed in this article provide evidence that US guidance of paracentesis is associated with a higher rate of successful aspiration of fluid, a lower rate of hemorrhagic complications and no higher risk of infection as compared to the landmark technique. Although not clearly demonstrated in the literature, direct imaging of a patient's anatomy prior to needle puncture may decrease the incidence of perforation of organs, like bowel, bladder, liver or spleen. In the current climate of advocating for increased safety for patients undergoing invasive procedures in the hospital, US guidance of paracentesis should be definitively included in this campaign. This is reinforced as the blind landmark technique has been associated with a higher risk of complications and mortality, as well as increased hospital length of stay and charges for the patient. Clinicians caring for patients with suspected and confirmed ascites should strongly consider adding bedside US to their skill set to improve the initial diagnosis and evaluation of these patients, as well as to facilitate the performance of the paracentesis procedure.

\section{References}

[1] Runyon, B.A. (1998) Management of Adult Patients with Ascites Caused by Cirrhosis. Hepatology, 27, $264-272$. http://dx.doi.org/10.1002/hep.510270139

[2] Gines, P., Cardenas, A., Arroyo, V., et al. (2004) Current Concepts: Management of Cirrhosis and Ascites. The New England Journal of Medicine, 350, 1646-1654. http://dx.doi.org/10.1056/NEJMra035021

[3] Chinnock, B., Afarian, H., Minnigan, H., et al. (2008) Physician Clinical Impression Does Not Rule out Spontaneous Bacterial Peritonitis in Patients Undergoing Emergency Department Paracentesis. Annals of Emergency Medicine, 52, 268-273. http://dx.doi.org/10.1016/j.annemergmed.2008.02.016

[4] World Gastroenterology Organization (2014) WGO Practice Guideline: Condition: Management of Ascites Complicating Cirrhosis in Adults. 
http://www.worldgastroenterology.org/assets/downloads/en/pdf/guidelines/14_management_ascites_en.pdf

[5] Sakai, H., Sheer, T., Mendler, M., et al. (2005) Choosing the Location for Non-Image Guided Abdominal Paracentesis. Liver International, 25, 984-986. http://dx.doi.org/10.1111/j.1478-3231.2005.01149.X

[6] Longo, D., Fauci, A., Kasper, D., et al. (2013) Harrison’s Manual of Medicine. 18th Edition, McGraw-Hill Education Medical, New York.

[7] Wiese, S., Mortensen, C. and Bendtsen, F. (2011) Few Complications after Paracentesis in Patient with Cirrhosis and Refractory Ascites. Danish Medical Bulletin, 58, A4212.

[8] Runyon, B. (1986) Paracentesis of Ascitic Fluid: A Safe Procedure. Archives of Internal Medicine, 146, $2259-2261$. http://dx.doi.org/10.1001/archinte.1986.00360230201029

[9] Mallory, A. and Schaefer, J. (1978) Complications of Diagnostic Paracentesis in Patients with Liver Disease. JAMA, 239, 628-630. http://dx.doi.org/10.1001/jama.1978.03280340048020

[10] Mercaldi, C.J. and Lanes, S.F. (2013) Ultrasound Guidance Decreases Complications and Improves the Cost of Care among Patients Undergoing Thoracentesis and Paracentesis. Chest, 143, 532-538. http://dx.doi.org/10.1378/chest.12-0447

[11] Irshad, A., Ackerman, S., Anis, M., et al. (2009) Can the Smallest Depth of Ascitic Fluid on Sonograms Predict the Amount of Drainable Fluid? Journal of Clinical Ultrasound, 37, 440-444. http://dx.doi.org/10.1002/jcu.20616

[12] McGahan, J., Anderson, M. and Walter, J. (1986) Portable Real-Time Sonographic and Needle Guidance Systems for Aspiration and Drainage. American Journal of Roentgenology, 147, 1241-1246. http://dx.doi.org/10.2214/ajr.147.6.1241

[13] Goldberg, B.B., Goodman, G.A. and Clearfield, H.R. (1970) Evaluation of Ascites by Ultrasound. Radiology, 96, 1522. http://dx.doi.org/10.1148/96.1.15

[14] Von Kuenssberg Jehle, D., Stiller, G. and Wagner, D. (2003) Sensitivity in Detecting Free Intraperitoneal Fluid with the Pelvic Views of the FAST Exam. The American Journal of Emergency Medicine, 21, 476-478. http://dx.doi.org/10.1016/S0735-6757(03)00162-1

[15] Branney, S., Wolfe, R., Moore, R., et al. (1995) Quantitative Sensitivity of Ultrasound in Detecting Free Intraperitoneal Fluid. The Journal of Trauma: Injury, Infection and Critical Care, 39, 375-380. http://dx.doi.org/10.1097/00005373-199508000-00032

[16] Williams, J. and Simel, D. (1992) The Rational Clinical Examination. Does This Patient Have Ascites? How to Divine Fluid in the Abdomen. JAMA, 267, 2645-2648. http://dx.doi.org/10.1001/jama.1992.03480190087038

[17] Tibbles, C.D. and Porcaro, W. (2004) Procedural Applications of Ultrasound. Emergency Medicine Clinics of North America, 22, 797-815. http://dx.doi.org/10.1016/j.emc.2004.04.010

[18] Moore, C. and Copel, J. (2011) Point-of-Care Ultrasonography. The New England Journal of Medicine, 364, 749-757. http://dx.doi.org/10.1056/NEJMra0909487

[19] American College of Emergency Physicians (2008) Policy Statement: Emergency Ultrasound Guidelines. http://www.acep.org/workarea/DownloadAsset.aspx?id=32878

[20] Tirado, A., Wu, T., Noble, V.E., et al. (2013) Ultrasound-Guided Procedures in the Emergency Department-Diagnostic and Therapeutic Asset. Emergency Medicine Clinics of North America, 31, 117-149. http://dx.doi.org/10.1016/j.emc.2012.09.009

[21] Ross, G., Kessler, H., Clair, M., et al. (1989) Sonographically Guided Paracentesis for Palliation of Symptomatic Malignant Ascites. American Journal of Roentgenology, 153, 1309-1311. http://dx.doi.org/10.2214/ajr.153.6.1309

[22] Bard, C., Lafortune, M. and Breton, G. (1986) Ascites: Ultrasound Guidance or Blind Paracentesis? Canadian Medical Association Journal, 135, 209-210.

[23] Wong, C., Holroyd-Leduc, J., Thorpe, K., et al. (2008) Does This Patient Have Bacterial Peritonitis or Portal Hypertension? How Do I Perform a Paracentesis and Analyze the Results? JAMA, 299, 1166-1178. http://dx.doi.org/10.1001/jama.299.10.1166

[24] Grabau, C.M., Crago, S.F., Hoff, L.K., et al. (2004) Performance Standards for Therapeutic Abdominal Paracentesis. Hepatology, 40, 484-488. http://dx.doi.org/10.1002/hep.20317

[25] McGibbon, A., Chen, G., Peltekian, K., et al. (2007) An Evidence-Based Manual for Abdominal Paracentesis. Digestive Diseases and Sciences, 52, 3307-3315. http://dx.doi.org/10.1007/s10620-007-9805-5

[26] McVay, P.A. and Toy, P.T.C.Y. (1991) Lack of Increased Bleeding after Paracentesis and Thoracentesis in Patients with Mild Coagulation Abnormalities. Transfusion, 31, 164-171. http://dx.doi.org/10.1046/j.1537-2995.1991.31291142949.x

[27] Pache, I. and Bilodeau, M. (2005) Severe Haemorrhage Following Abdominal Paracentesis for Paracentesis in Patients 
with Liver Disease. Alimentary Pharmacology \& Therapeutics, 21, 525-529. http://dx.doi.org/10.1111/j.1365-2036.2005.02387.x

[28] Degottardi, A., Thevenot, T., Spahr, L., et al. (2009) Risk of Complications after Abdominal Paracentesis in Cirrhotic Patients: A Prospective Study. Clinical Gastroenterology and Hepatology, 7, 906-909. http://dx.doi.org/10.1016/j.cgh.2009.05.004

[29] Koffel, K.K. and Reed, J.S. (1986) The Technique of Abdominal Paracentesis. The Journal of Critical Illness, 1, 4550.

[30] Hatch, N. and Wu, T. (2014) Preface: Ultrasound: Part 1. Advanced Ultrasound Procedures: Review Article. Critical Care Clinics, 30, 305-329. http://dx.doi.org/10.1016/j.ccc.2013.10.005

[31] Sekiguchi, H., Suzuki, J. and Daniels, C. (2013) Making Paracentesis Safer: A Proposal for the Use of Bedside Ultrasonography to Prevent a Fatal Complication. Chest, 143, 1136-1139. http://dx.doi.org/10.1378/chest.12-0871

[32] Epstein, J., Aroroa, A. and Ellis, H. (2004) Surface Anatomy of the Inferior Epigastric Artery in Relation to Laparoscopic Injury. Clinical Anatomy, 17, 400-408. http://dx.doi.org/10.1002/ca.10192

[33] Oelsner, D., Caldwell, S., Coles, M., et al. (1998) Subumbilical Midline Vascularity of the Abdominal Wall in Portal Hypertension Observed at Laparoscopy. Gastrointestinal Endoscopy, 47, 388-390.

[34] Shaheen, N. and Grimm, I. (1996) Comparison of the Caldwell Needle/Cannula with Angiocath Needle in Large Volume Paracentesis. The American Journal of Gastroenterology, 91, 1731-1733.

[35] Runyon, M.S. and Marx, J.A. (2014) Peritoneal Procedures. In: Roberts, J.R., Hedges, J.R., Custalow, C.B., et al., Eds., Roberts and Hedges' Clinical Procedures in Emergency Medicine, 6th Edition, Elsevier Saunders, Philadephia, 852872.

[36] Nazeer, S.R., Dewbre, H. and Miller, A.H. (2005) Ultrasound-Assisted Paracentesis Performed by Emergency Physicians vs the Traditional Technique: A Prospective, Randomized Study. American Journal of Emergency Medicine, 23, 363-367. http://dx.doi.org/10.1016/j.ajem.2004.11.001

[37] Webster, S.T., Brown, K.L., Lucey, M.R., et al. (1996) Hemorrhagic Complications of Large Volume Abdominal Paracentesis. The American Journal of Gastroenterology, 91, 366-368.

[38] Arnold, C., Haag, K., Blum, H.E. and Rossle, M. (1997) Acute Hemoperitoneum after Large-Volume Paracentesis. Gastroenterology, 113, 978-982. http://dx.doi.org/10.1016/S0016-5085(97)70210-5

[39] Qureshi, W.A., Harshfield, D., Shah, H., et al. (1992) An Unusual Complication of Paracentesis. The American Journal of Gastroenterology, 87, 1209-1211.

[40] Aagaard, J., Jensen, L.I., Sørensen, T.I., et al. (1982) Recanalized Umbilical Vein in Portal Hypertension. American Journal of Roentgenology, 139, 1107-1110. http://dx.doi.org/10.2214/ajr.139.6.1107

[41] Patel, P.A., Ernst, F.R. and Gunnarsson, C.L. (2012) Evaluation of Hospital Complications and Costs Associated with Using Ultrasound Guidance during Abdominal Paracentesis Procedures. Journal of Medical Economics, 15, 1-7. http://dx.doi.org/10.3111/13696998.2011.628723

[42] Cervini, P., Hesley, G.K., Thompson, R.L., et al. (2010) Incidence of Infectious Complications after an UltrasoundGuided Intervention. American Journal of Roentgenology, 195, 846-850. http://dx.doi.org/10.2214/AJR.09.3168 
Scientific Research Publishing (SCIRP) is one of the largest Open Access journal publishers. It is currently publishing more than 200 open access, online, peer-reviewed journals covering a wide range of academic disciplines. SCIRP serves the worldwide academic communities and contributes to the progress and application of science with its publication.

Other selected journals from SCIRP are listed as below. Submit your manuscript to us via either submit@scirp.org or Online Submission Portal.
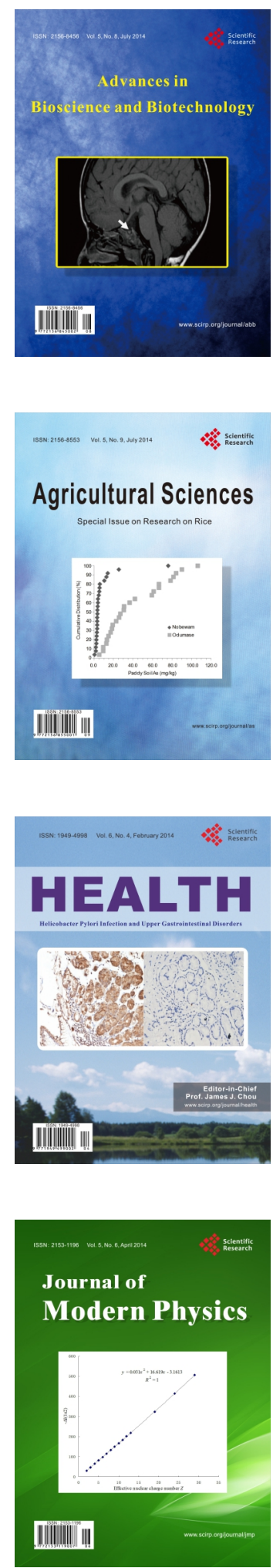
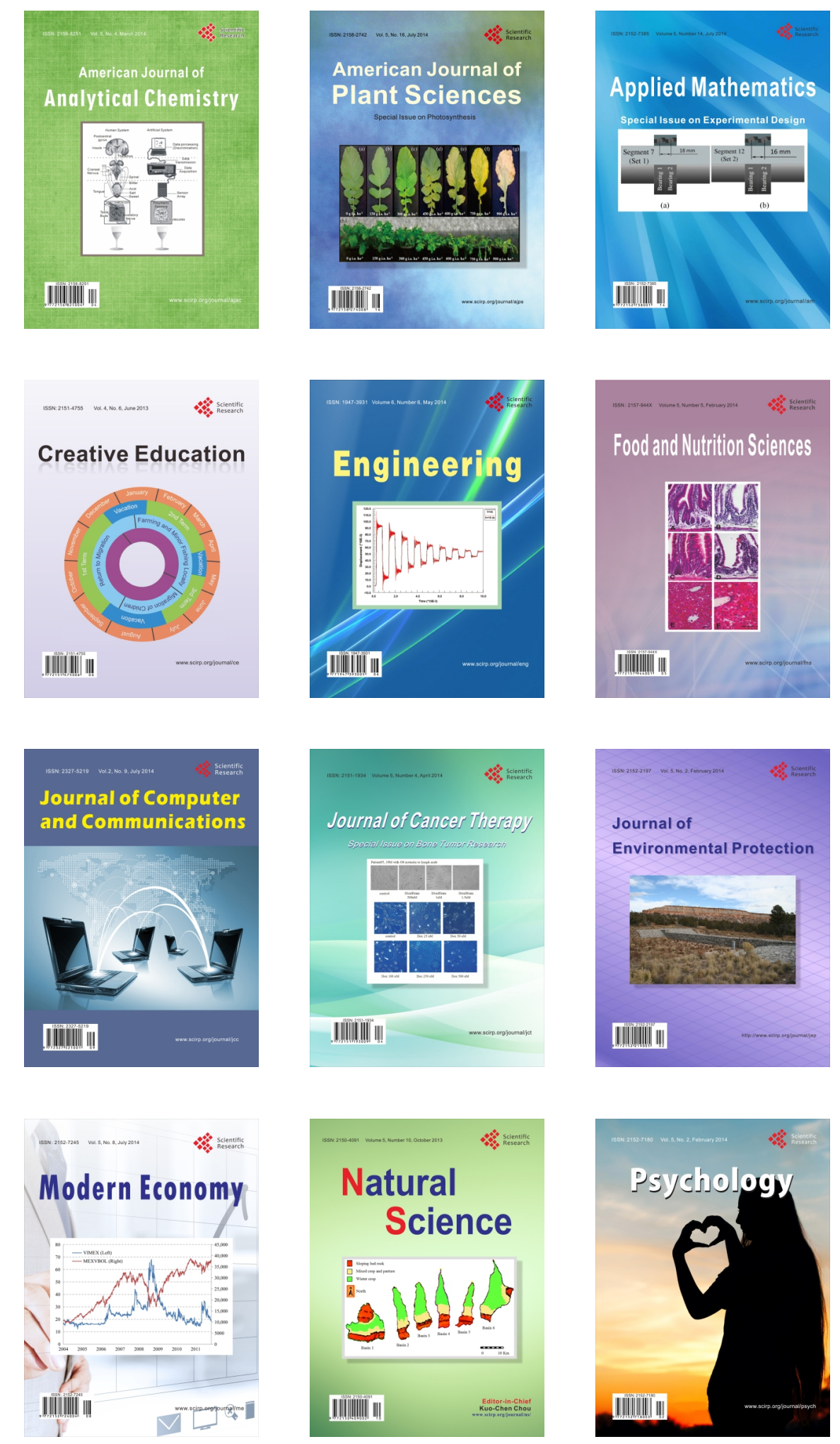